\title{
SIEGEL MODULAR FORMS OF GENUS 2 AND LEVEL 2: COHOMOLOGICAL COMPUTATIONS AND CONJECTURES
}

\author{
JONAS BERGSTRÖM, CAREL FABER, AND GERARD VAN DER GEER
}

\section{INTRODUCTION}

In this paper we study the cohomology of certain local systems on moduli spaces of principally polarized abelian surfaces with a level 2 structure that corresponds to prescribing a number of Weierstrass points in case the abelian surface is the Jacobian of a curve of genus 2. These moduli spaces are defined over $\mathbb{Z}[1 / 2]$ and we can calculate the trace of Frobenius on the alternating sum of the étale cohomology groups of these local systems by counting the number of pointed curves of genus 2 with a prescribed number of Weierstrass points that separately or taken together are defined over the given finite field. This cohomology is intimately related to vector-valued Siegel modular forms. Two of the present authors carried out this scheme for local systems on the moduli space $\mathcal{A}_{2}$ of level 1 in [11. This provided new information on Siegel modular forms and led for example to a precise formulation of a conjecture of Harder about congruences between genus 1 and genus 2 modular forms and also to experimental evidence supporting it, cf. [16, 13].

Here we extend this scheme to level 2, where new phenomena appear. In order to be able to extract information on Siegel modular forms we must subtract the contributions to the cohomology from the boundary, that is, the Eisenstein cohomology, and the endoscopic contributions. We determine the contribution of the Eisenstein cohomology together with its $\mathbb{S}_{6}$-action for the full level 2 structure and on the basis of our computations we make precise conjectures on the endoscopic contribution. We also make a prediction about the existence of a vector-valued analogue of the Saito-Kurokawa lift. Assuming these conjectures that are based on ample numerical evidence, we obtain the traces of the Hecke-operators $T(p)$ for $p \leq 37$ on the remaining spaces of 'genuine' Siegel modular forms. We present a number of examples of 1-dimensional spaces of eigenforms where these traces coincide with the Hecke eigenvalues to illustrate this. We hope that the experts on lifting and on endoscopy will be able to prove our conjectures.

Acknowledgement. We thank R. Tsushima for sending us a program computing the dimension of the space of Siegel modular cusp forms of the group $\Gamma_{2}\left(w^{1}\right)$, used in Section 9. We also thank S. Kudla for correspondence and

1991 Mathematics Subject Classification. 11F46, 11G18, 14G35, 14J15, $20 \mathrm{~B} 25$. 
P. Deligne for comments on an earlier version. We also thank S. Böcherer and R. Schulze-Pillot for pointing out their work on Yoshida-type liftings and G. Harder for discussions on congruences. C.F. is supported by the Swedish Research Council (grant 622-2003-1123) and the Göran Gustafsson Foundation for Research in Natural Sciences and Medicine.

\section{The Moduli Spaces $\mathcal{A}_{2}\left(w^{n}\right)$}

Let $\mathcal{M}_{2}$ be the moduli space of curves of genus 2 and $\mathcal{A}_{2}$ the moduli space of principally polarized abelian surfaces. These are Deligne-Mumford stacks defined over $\operatorname{Spec}(\mathbb{Z})$ and by the Torelli map we can view $\mathcal{M}_{2}$ as an open substack of $\mathcal{A}_{2}$. A curve of genus 2 admits a unique morphism of degree 2 to $\mathbb{P}^{1}$ and its ramification points are called the Weierstrass points. If the element 2 is invertible on the base there are 6 Weierstrass points. We now can look at covers of $\mathcal{M}_{2}$, namely, for $0 \leq n \leq 6$ we consider the stack $\mathcal{M}_{2}\left(w^{n}\right)$ which is the moduli space of pairs $\left(C,\left(r_{1}, \ldots, r_{n}\right)\right)$ of curves of genus 2 together with $n$ ordered Weierstrass points. These are Deligne-Mumford stacks defined over $\operatorname{Spec}(\mathbb{Z}[1 / 2])$.

Let $\left(C, r_{1}, \ldots, r_{6}\right)$ be a genus 2 curve with its six numbered Weierstrass points. A Weierstrass point $r_{1}$ defines an embedding of $C$ into its Jacobian $\operatorname{Jac}(C)$ given by $q \mapsto q-r_{1}$. This provides us with a set of 5 points of order 2 of $\operatorname{Jac}(C)$, namely, $\left\{r_{i}-r_{1}: i=2, \ldots, 6\right\}$. The non-zero points of order 2 on $\operatorname{Jac}(C)$ correspond bijectively to the pairs $\left\{r_{i}, r_{j}\right\}$ with $i \neq j$.

By associating to a (decorated) curve its (decorated) Jacobian we have an embedding

$$
\mathcal{M}_{2}\left(w^{6}\right) \hookrightarrow \mathcal{A}_{2}[2]
$$

where $\mathcal{A}_{2}[2]$ is the moduli space of principally polarized abelian surfaces $X$ with a full level 2-structure, that is, an isomorphism of the kernel $X[2]$ of multiplication $2_{X}$ by 2 on $X$ with a fixed symplectic module $\left((\mathbb{Z} / 2)^{4},\langle\rangle,\right)$. The symmetric group $\mathbb{S}_{6}$ acts on $\mathcal{M}_{2}\left(w^{6}\right)$ and the group $\operatorname{GSp}(4, \mathbb{Z} / 2)$ acts on $\mathcal{A}_{2}[2]$ and the embedding defines an isomorphism of $\mathbb{S}_{6}$ with $\operatorname{GSp}(4, \mathbb{Z} / 2)$ which we will make explicit below. Let us identify $\operatorname{GSp}(4, \mathbb{Z} / 2)$ with $\mathbb{S}_{6}$ under this isomorphism and define the quotient stacks

$$
\mathcal{A}_{2}\left(w^{n}\right):=\mathcal{A}_{2}[2] / \mathbb{S}_{6-n},
$$

where $\mathbb{S}_{6-n}$ is the subgroup of $\mathbb{S}_{6}$ fixing $\{1, \ldots, n\}$ pointwise. Note that we have inclusions $\mathcal{M}_{2}\left(w^{n}\right) \hookrightarrow \mathcal{A}_{2}\left(w^{n}\right)$, and equalities $\mathcal{A}_{2}\left(w^{0}\right)=\mathcal{A}_{2}$ and $\mathcal{A}_{2}\left(w^{6}\right)=\mathcal{A}_{2}[2]$.

Let $U$ be a symplectic space of dimension 4 over $\mathbb{Z} / 2$ with basis $e_{1}, e_{2}, f_{1}, f_{2}$ and with symplectic form $\langle$,$\rangle such that \left\langle e_{i}, e_{j}\right\rangle=\left\langle f_{i}, f_{j}\right\rangle=0$ and $\left\langle e_{i}, f_{j}\right\rangle=$ $\delta_{i j}$ (Kronecker delta). One observes that $U$ contains six (maximal) sets $V_{i}$ $(i=1, \ldots, 6)$ of five vectors $u_{j} \in U-\{0\}$ with $\left\langle u_{j}, u_{k}\right\rangle=1$ for $j \neq k$. For instance, one such set is $\left\{e_{1}, f_{1}, e_{1}+f_{1}+f_{2}, e_{1}+e_{2}+f_{1}, e_{1}+e_{2}+f_{1}+f_{2}\right\}$. We have $\#\left(V_{i} \cap V_{j}\right)=1$ if $i \neq j$. The action of $\operatorname{GSp}(4, \mathbb{Z} / 2)$ on these six sets $V_{i}$ defines an isomorphism of $\operatorname{GSp}(4, \mathbb{Z} / 2)$ with $\mathbb{S}_{6}$. A Weierstrass point $r_{i}$ 
on a curve of genus 2 determines a set of 5 points $r_{j}-r_{i}(j \neq i)$ of order 2 and they satisfy $\left\langle r_{j}-r_{i}, r_{k}-r_{i}\right\rangle=1$ for $j \neq k$.

Consider the inverse image $\Gamma_{2}\left(w^{n}\right)$ under $\operatorname{Sp}(4, \mathbb{Z}) \rightarrow \operatorname{Sp}(4, \mathbb{Z} / 2) \rightarrow \mathbb{S}_{6}$ of a subgroup $\mathbb{S}_{6-n}$ of $\mathbb{S}_{6}$ fixing the set $V_{i}$ for each $i$ between 1 and $n$. Then the orbifold $\Gamma_{2}\left(w^{n}\right) \backslash \mathcal{H}_{2}$, with $\mathcal{H}_{2}$ the Siegel upper half space of degree 2, is the complex fibre of the moduli stack $\mathcal{A}_{2}\left(w^{n}\right)$. By $\Gamma_{2}[2]=\Gamma_{2}\left(w^{6}\right)$ we shall mean the kernel of $\operatorname{Sp}(4, \mathbb{Z}) \rightarrow \operatorname{Sp}(4, \mathbb{Z} / 2)$.

\section{LOCAL Systems}

Let $\pi: \mathcal{X} \rightarrow \mathcal{A}_{2}$ denote the universal abelian surface. The moduli space $\mathcal{A}_{2}$ carries natural local systems $\mathbb{V}_{l, m}$ indexed by the pairs $(l, m)$ of integers with $l \geq m \geq 0$ that correspond to irreducible representations of $\operatorname{GSp}(4)$, cf. [11. The local system $\mathbb{V}_{1,0}$ is the one defined by $\mathbb{V}:=R^{1} \pi_{*} \mathbb{Q}_{\ell}$ and $\mathbb{V}_{l, m}$ is of weight $l+m$ and occurs 'for the first time' in $\operatorname{Sym}^{l-m}(\mathbb{V}) \otimes \operatorname{Sym}^{m}\left(\wedge^{2} \mathbb{V}\right)$. When $l>m>0$ the local system is called regular. By pullback to $\mathcal{A}_{2}\left(w^{n}\right)$ we obtain local systems which we will denote by the same symbol $\mathbb{V}_{l, m}$. We are interested in the cohomology (with compact support) of these local systems, more precisely in the motivic Euler characteristic

$$
e_{c}\left(\mathcal{A}_{2}\left(w^{n}\right), \mathbb{V}_{l, m}\right)=\sum_{i=0}^{6}(-1)^{i}\left[H_{c}^{i}\left(\mathcal{A}_{2}\left(w^{n}\right), \mathbb{V}_{l, m}\right)\right],
$$

where this expression is taken in the Grothendieck group of an appropriate category (for instance the category of mixed Hodge structures). Note that this cohomology is zero if $l+m$ is odd and thus we will from now on only consider the case when $l+m$ is even.

For any $\mathcal{A}$, among the moduli spaces considered, there is a natural map $H_{c}^{*}\left(\mathcal{A}, \mathbb{V}_{l, m}\right) \rightarrow H^{*}\left(\mathcal{A}, \mathbb{V}_{l, m}\right)$ whose image is called the inner cohomology and denoted by $H_{!}^{*}\left(\mathcal{A}, \mathbb{V}_{l, m}\right)$. By work of Faltings and Chai one knows that for regular $\lambda$, the cohomology groups $H_{!}^{i}\left(\mathcal{A}, \mathbb{V}_{l, m}\right)$ vanish for $i \neq 3$ (see [9], Cor. to Thm. 7, p. 84 and [10], p. 233-7). Moreover, one knows that $H^{3}\left(\mathcal{A}, \mathbb{V}_{l, m}\right)$ (resp. $\left.H_{c}^{3}\left(\mathcal{A}, \mathbb{V}_{l, m}\right)\right)$ carry mixed Hodge structures of weights $\geq l+m+3$ (resp. $\leq l+m+3)$ and that $H_{!}^{3}\left(\mathcal{A}, \mathbb{V}_{l, m}\right)$ carries a pure Hodge structure with Hodge filtration

$$
\text { (0) } \subset F^{l+m+3} \subset F^{l+2} \subset F^{m+1} \subset F^{0}=H_{!}^{3}\left(\mathcal{A}, \mathbb{V}_{l, m}\right) .
$$

The first step in this Hodge filtration is connected to Siegel modular forms by the isomorphism

$$
F^{l+m+3} \cong S_{l-m, m+3}\left(\Gamma_{2}\left(w^{n}\right)\right),
$$

where $S_{l-m, m+3}\left(\Gamma_{2}\left(w^{n}\right)\right)$ is the complex vector space of vector-valued cusp forms of weight $(l-m, m+3)$ on the group $\Gamma_{2}\left(w^{n}\right)$, cf. [10, Thm. 5.5, see also 15, Thm. 17. By a Siegel modular form of weight $(j, k)$ we mean a vector-valued function on the Siegel upper half space that transforms with 
the factor of automorphy:

$$
\operatorname{Sym}^{j}(c \tau+d) \operatorname{det}(c \tau+d)^{k}
$$

for $(a, b ; c, d) \in \operatorname{Sp}(4, \mathbb{Z})$ or in a subgroup $\Gamma_{2}\left(w^{n}\right)$. Thus, the cohomology of the moduli spaces considered is closely connected to Siegel modular forms of the corresponding groups. In [11] two of the three present authors studied the cohomology in the case of level 1 (i.e. on $\mathcal{A}_{2}$ ) using counts of curves of genus 2 of compact type defined over finite fields (cf. also [15]).

Recall that the Eichler-Shimura theorem says (cf. [8]) that for the local system $\mathbb{V}_{k}:=\operatorname{Sym}^{k}(\mathbb{V})$ (with $\mathbb{V}:=R^{1} \pi_{*} \mathbb{Q}_{l}$ ), on the moduli space $\mathcal{A}_{1}$ of elliptic curves with universal family $\pi: \mathcal{X}_{1} \rightarrow \mathcal{A}_{1}$, one has for even $k \geq 2$,

$$
\begin{array}{r}
-e_{c}\left(\mathcal{A}_{1}, \mathbb{V}_{k}\right)=S[\operatorname{SL}(2, \mathbb{Z}), k+2]+1, \\
-e\left(\mathcal{A}_{1}, \mathbb{V}_{k}\right)=S[\operatorname{SL}(2, \mathbb{Z}), k+2]+L^{k+1},
\end{array}
$$

where we from now on denote by $L:=h^{2}\left(\mathbb{P}^{1}\right)$ the Tate motive of weight 2 and by $S[\mathrm{SL}(2, \mathbb{Z}), k+2]$ the motive of cusp forms of weight $k+2$ of $\mathrm{SL}(2, \mathbb{Z})$ as constructed by Scholl, cf. [20] (see also [7] for an alternative construction). For $k=0$ one can use the same formulas if one puts $S[\operatorname{SL}(2, \mathbb{Z}), 2]:=-L-1$.

\section{The Eisenstein COHOMOlOgy}

The compactly supported cohomology has a natural map to the usual cohomology and the kernel is called the Eisenstein cohomology. The corresponding motivic Euler characteristic is denoted by $e_{\text {Eis }}\left(\mathcal{A}, \mathbb{V}_{l, m}\right)$. By the full Eisenstein cohomology we mean the difference between the compactly supported and the usual cohomology, with corresponding Euler characteristic,

$$
e_{\text {Eis }^{\mathrm{f}}}\left(\mathcal{A}, \mathbb{V}_{l, m}\right):=e_{c}\left(\mathcal{A}, \mathbb{V}_{l, m}\right)-e\left(\mathcal{A}, \mathbb{V}_{l, m}\right) .
$$

For example, for genus 1 we have by Eichler-Shimura $e_{\text {Eis }}\left(\mathcal{A}_{1}, \mathbb{V}_{k}\right)=-1$, and for the full Eisenstein cohomology $e_{\operatorname{Eis}^{\mathrm{f}}}\left(\mathcal{A}_{1}, \mathbb{V}_{k}\right)=L^{k+1}-1$.

Remark 4.1. The full Eisenstein cohomology is anti-invariant under Poincaré duality and $e_{\text {Eis }}\left(\mathcal{A}, \mathbb{V}_{l, m}\right)$ determines the full Eisenstein cohomology by antisymmetrizing. The converse also holds by considerations of weights if $\lambda$ is regular, cf. [19].

We shall write $\Gamma(2)$ for the full level 2 congruence subgroup of $\operatorname{SL}(2, \mathbb{Z})$ and $\Gamma_{0}(N)$ for the congruence subgroup of matrices $(a, b ; c, d)$ with $N \mid c$. For any of these groups $\Gamma$, we will write $S_{k}(\Gamma)$ and $S[\Gamma, k]$ for the space, respectively the motive, of cusp forms of $\Gamma$ of weight $k$. The motive $S[\Gamma, k]$ has a Hodge realization that decomposes as $S_{k}(\Gamma) \oplus \bar{S}_{k}(\Gamma)$. Let $S_{k}(\Gamma)^{\text {new }}$ denote the subset of newforms in $S_{k}(\Gamma)$, and $S[\Gamma, k]^{\text {new }}$ the corresponding motive, constructed in [20]. 
Theorem 4.2. For regular pairs $(l, m)$ the Eisenstein cohomology of the local system $\mathbb{V}_{l, m}$ on the moduli space $\mathcal{A}_{2}[2]$ is given by

$$
\begin{aligned}
15 \operatorname{dim} S_{l-m+2}(\Gamma(2))- & 15 \operatorname{dim} S_{l+m+4}(\Gamma(2)) L^{m+1}+ \\
+ & 15\left\{\begin{array}{l}
S[\Gamma(2), m+2]+3 \\
-S[\Gamma(2), l+3]
\end{array} \text { if } m \text { if } m \text { oven }\right. \text { odd. }
\end{aligned}
$$

This can be proved as in [14] using the BGG-complex of Faltings-Chai (see [10]), by first computing $e_{\operatorname{Eis}^{\mathrm{f}}}\left(\mathcal{A}_{2}[2], \mathbb{V}_{l, m}\right)$ and then deducing $e_{\text {Eis }}\left(\mathcal{A}_{2}[2], \mathbb{V}_{l, m}\right)$, see Remark 4.1. If the pair $(l, m)$ is not regular we still expect the formula to hold as long as we put $S[\Gamma(2), 2]:=-L-1$ in case $m=0$ and $\operatorname{dim} S_{2}(\Gamma(2)):=-1$ in case $l=m$.

The factors 15 in the formula come from the fact that the Satake compactification of $\mathcal{A}_{2}[2]$ has 15 one-dimensional and 15 zero-dimensional boundary components.

The group $\mathbb{S}_{6}$ acts on $\mathcal{A}_{2}[2]$ and this induces an action on the Eisenstein cohomology of a local system. We can decompose this piece of the cohomology into irreducible representations for $\mathbb{S}_{6}$. Note that we can identify $S_{k}(\Gamma(2))$ with $S_{k}\left(\Gamma_{0}(4)\right)$ via the map $f(z) \mapsto f(2 z)$ and the corresponding motive can be split as

$$
S\left[\Gamma_{0}(4), k\right]=S\left[\Gamma_{0}(4), k\right]^{\text {new }}+2 S\left[\Gamma_{0}(2), k\right]^{\text {new }}+3 S[\mathrm{SL}(2, \mathbb{Z}), k] .
$$

We need some notation concerning representations of $\mathbb{S}_{6}$. Recall that the irreducible representations are in natural bijective correspondence with the partitions of the number 6 . We shall write the representation corresponding to the partition $p$ as $s[p]$. Let us write

$$
\begin{aligned}
& A:=s\left[3,1^{3}\right]+s\left[2,1^{4}\right]=\operatorname{Ind}\left(s[2] \otimes s\left[1^{4}\right]\right), \\
& B:=s[4,2]+s[3,2,1]+s\left[2^{3}\right]=\operatorname{Ind}\left(s[2] \otimes s\left[2^{2}\right]\right), \\
& C:=s[6]+s[5,1]+s[4,2]=\operatorname{Ind}(s[2] \otimes s[4]),
\end{aligned}
$$

where Ind denotes induction from $\mathbb{S}_{2} \times \mathbb{S}_{4} \subset \mathbb{S}_{6}$ to $\mathbb{S}_{6}$. We can view $\mathbb{S}_{2} \times \mathbb{S}_{4}$ as the stabilizer of a fixed element in $U-\{0\}$ for the action of $\mathbb{S}_{6} \cong \operatorname{GSp}(4, \mathbb{Z} / 2)$. Moreover, the stabilizer of a 2-dimensional totally isotropic subspace of $U$ corresponds to the stabilizer $H$ of (say) (12)(34)(56) for the conjugation action of $\mathbb{S}_{6}$. This group is isomorphic to the semi-direct product $\mathbb{S}_{3} \ltimes$ $(\mathbb{Z} / 2)^{3}$. The irreducible representations of $H$ are given by pairs of irreducible representations, one of $(\mathbb{Z} / 2)^{3}$, taken up to the action of $\mathbb{S}_{3}$, and one of its stabilizer subgroup in $\mathbb{S}_{3}$. We put

$$
\begin{aligned}
& A^{\prime}:=s\left[4,1^{2}\right]+s\left[3^{2}\right]=\operatorname{Ind}_{H}^{\mathbb{S}_{6}}\left(s\left[1^{3}\right] \otimes \mathrm{id}\right), \\
& B^{\prime}:=s[5,1]+s[4,2]+s[3,2,1]=\operatorname{Ind}_{H}^{\mathbb{S}_{6}}(s[2,1] \otimes \mathrm{id}), \\
& C^{\prime}:=s[6]+s[4,2]+s\left[2^{3}\right]=\operatorname{Ind}_{H}^{\mathbb{S}_{6}}(s[3] \otimes \mathrm{id}) .
\end{aligned}
$$

We note that $\operatorname{dim} A=\operatorname{dim} A^{\prime}=\operatorname{dim} C=\operatorname{dim} C^{\prime}=15$ while $\operatorname{dim} B=$ $\operatorname{dim} B^{\prime}=30$. 
Notation 4.3. Define $\tau_{N, k}:=\operatorname{dim} S_{k}\left(\Gamma_{0}(N)\right)^{\text {new }}$.

Theorem 4.4. For regular pairs $(l, m)$ the contributions in Thm. 4.2 to the Eisenstein cohomology of the local system $\mathbb{V}_{l, m}$ can be decomposed under the action of $\mathbb{S}_{6}$ as follows. The term $15 \operatorname{dim} S_{k}\left(\Gamma_{0}(4)\right)$ with $k=l-m+2$ or $k=l+m+4$ decomposes as

$$
\tau_{4, k} \cdot A^{\prime}+\left(\tau_{2, k}+\tau_{1, k}\right) \cdot B^{\prime}+\tau_{1, k} \cdot C^{\prime}
$$

while the term $15 S\left[\Gamma_{0}(4), k\right]$ with $k=m+2$ or $k=l+3$ can be written as

$$
A \otimes S\left[\Gamma_{0}(4), k\right]^{\text {new }}+B \otimes S\left[\Gamma_{0}(2), k\right]^{\text {new }}+(B+C) \otimes S\left[\Gamma_{0}(1), k\right]
$$

and finally $15 \cdot 3 L^{0}=(B+C) L^{0}$.

For $l=m$ we conjecture that $15 \operatorname{dim} S_{2}\left(\Gamma_{0}(4)\right)$ decomposes as $-C^{\prime}$, and for $m=0$ that the term $15 S\left[\Gamma_{0}(4), 2\right]$ decomposes as $C \cdot(-L-1)$. The theorem can be proved by the method of [14] taking into account the action of $\mathbb{S}_{6}$ on the boundary components.

The $\mathbb{S}_{6}$-decomposition of the Eisenstein cohomology on $\mathcal{A}_{2}[2]$ allows one to deduce the formulas for $\mathcal{A}_{2}\left(w^{n}\right)$ for $0 \leq n \leq 6$. For example for $\mathcal{A}_{2}\left(w^{1}\right)$ and $\mathcal{A}_{2}\left(w^{3}\right)$ we find the following.

Corollary 4.5. For regular $(l, m)$ the Eisenstein cohomology of $\mathbb{V}_{l, m}$ on $\mathcal{A}_{2}\left(w^{1}\right)$ is given by

$\operatorname{dim} S_{l-m+2}\left(\Gamma_{0}(2)\right)-\operatorname{dim} S_{l+m+4}\left(\Gamma_{0}(2)\right) L^{m+1}+ \begin{cases}2(S[m+2]+1) & \text { if } m \text { even } \\ -2 S[l+3] & \text { if } m \text { odd } .\end{cases}$

Corollary 4.6. For regular $(l, m)$ the Eisenstein cohomology of $\mathbb{V}_{l, m}$ on $\mathcal{A}_{2}\left(w^{3}\right)$ is given by

$$
\begin{aligned}
& 4 \operatorname{dim} S_{l-m+2}\left(\Gamma_{0}(4)\right)-4 \operatorname{dim} S_{l+m+4}\left(\Gamma_{0}(4)\right) L^{m+1}+ \\
& + \begin{cases}3 S\left[\Gamma_{0}(1), m+2\right]+3 S\left[\Gamma_{0}(2), m+2\right]+S\left[\Gamma_{0}(4), m+2\right]+12 & \text { if } m \text { even } \\
-3 S\left[\Gamma_{0}(1), l+3\right]-3 S\left[\Gamma_{0}(2), l+3\right]-S\left[\Gamma_{0}(4), l+3\right] & \text { if } m \text { odd. }\end{cases}
\end{aligned}
$$

\section{Counting Points Over finite Fields}

Recall that the moduli space $\mathcal{A}_{2}[2]$ of principally polarized abelian surfaces with level 2 structure can be identified with the moduli space of tuples $\left(C, r_{1}, \ldots, r_{6}\right)$, where $C$ is either an irreducible genus 2 curve or a pair of genus 1 curves intersecting in one point, and where $\left(r_{1}, \ldots, r_{6}\right)$ is a 6 -tuple of marked Weierstrass points. In the case of two intersecting elliptic curves these are the points of order 2 on the two elliptic curves taking the intersection point as origin on both.

For an odd prime number $p$ we consider this moduli space over the field $\overline{\mathbb{F}}_{q}$, where $q$ is a power of $p$. Let $H_{e ́ t}^{i}$ denote the compactly supported $\ell$-adic étale cohomology. The natural action of $\mathbb{S}_{6}$ on $\mathcal{A}_{2}[2]$ induces a decomposition of $H_{e ́ t}^{i}\left(\mathcal{A}_{2}[2] \otimes \overline{\mathbb{F}}_{q}, \mathbb{V}_{l, m}\right)$ into pieces denoted $H_{e ́ t, \mu}^{i}\left(\mathcal{A}_{2}[2] \otimes \overline{\mathbb{F}}_{q}, \mathbb{V}_{l, m}\right)$, for each 
irreducible representation of $\mathbb{S}_{6}$ indexed by the partition $\mu$ of 6 . We wish to compute the trace of Frobenius $F_{q}$ on the Euler characteristic

$$
e_{e ́ t, \mu}\left(\mathcal{A}_{2}[2] \otimes \overline{\mathbb{F}}_{q}, \mathbb{V}_{l, m}\right):=\sum_{i}(-1)^{i} H_{e ́ t, \mu}^{i}\left(\mathcal{A}_{2}[2] \otimes \overline{\mathbb{F}}_{q}, \mathbb{V}_{l, m}\right) .
$$

The necessary information to compute this, for any partition $\mu$ and pair $(l, m)$, was found for all odd $q \leq 37$ with the aid of the computer. We indicate below how this was done.

We will denote by $k$ a finite field and by $k_{2}$ a degree 2 extension of $k$.

5.1. Irreducible curves of genus 2. Let $P_{2}(k) \subset k[x]$ be the set of of all square-free polynomials of degree 5 or 6 . Each element $f \in P_{2}(k)$ defines a curve $C_{f}$ of genus 2 defined by $y^{2}=f(x)$. For each $f \in P_{2}(k)$ and $k \in \mathcal{K}:=\left\{\mathbb{F}_{q}: 2 \nmid q, q \leq 37\right\}$ we computed the following: i) the number of points of $C_{f}$ defined over $k$; ii) the number of points of $C_{f}$ defined over $k_{2}$; iii) the fields of definition of all 6 ramification points of the canonical map $C_{f} \rightarrow \mathbb{P}^{1}$ given by $(x, y) \rightarrow x$.

For a partition $\nu$ of 6 let $P_{2}(\nu, k) \subset P_{2}(k)$ be the subset of polynomials $f$ defining curves $C_{f}$ which have fields of definition of their ramification points given by $\nu$. Using the Lefschetz trace formula we can now, for each pair of numbers $n_{1}, n_{2}$ and $k \in \mathcal{K}$, compute

$$
a\left(\mathcal{M}_{2}, \nu, n_{1}, n_{2}\right):=\sum_{f \in P_{2}(\nu, k)} a_{1}\left(C_{f}\right)^{n_{1}} \cdot a_{2}\left(C_{f}\right)^{n_{2}} /\left|\mathrm{GL}_{2}(k)\right|,
$$

where $a_{1}\left(C_{f}\right):=\operatorname{Tr}\left(F_{q}, H_{\text {ét }}^{1}\left(C_{f}\right)\right)$ and $a_{2}\left(C_{f}\right):=\operatorname{Tr}\left(F_{q}^{2}, H_{\text {ét }}^{1}\left(C_{f}\right)\right)$. Note that $\left|\mathrm{GL}_{2}(k)\right|$ is the number of $k$-isomorphisms between the curves of $P_{2}(\nu, k)$.

5.2. Pairs of elliptic curves. Similarly, let $P_{1}(k) \subset k[x]$ consist of all square-free polynomials $f(x) \in k[x]$ of degree 3 and let $\mathcal{K}^{\prime}:=\left\{\mathbb{F}_{q}, \mathbb{F}_{q^{2}}\right.$ : $2 \nmid q, q \leq 37\}$ be a collection of finite fields. Each element of $P_{1}(k)$ defines an elliptic curve $C_{f}$ given by $y^{2}=f(x)$ with $x=\infty$ as origin. For each element $k \in \mathcal{K}^{\prime}$ and $f \in P_{1}(k)$ with corresponding curve $C_{f}$ we computed the following: i) the number of points of $C_{f}$ defined over $k$; ii) the fields of definition of the 3 affine ramification points of the map $C_{f} \rightarrow \mathbb{P}^{1}$ given by $(x, y) \rightarrow x$.

To get the analogue of (5.1) for the pairs of elliptic curves joined at the origin, we should sum over all possibilities of distributing the ramification points and the marked points (which correspond to the monomials $\left.a_{1}\left(C_{f}\right)^{n_{1}} a_{2}\left(C_{f}\right)^{n_{2}}\right)$ on the two elliptic curves. Let us define $a\left(\mathcal{A}_{1,1}, \nu, n_{1}, n_{2}\right)$ to be the sum, over all ordered choices of partitions $\rho$ and $\sigma$ of 3 such that $\nu=\rho+\sigma$ and integers $m_{1} \leq n_{1}$ and $m_{2} \leq n_{2}$, of the following. We put $I_{k}^{\prime}:=\left|\mathrm{GL}_{2}(k)\right| /(|k|+1)$, which is the number of $k$-isomorphisms between the curves of $P_{1}(k)$, and we divide into two cases according to if there is an automorphism which interchanges the two elliptic curves or not. 
Case i) If $\rho \neq \sigma$ or $\left(m_{1}, m_{2}\right) \neq\left(n_{1}-m_{1}, n_{2}-m_{2}\right)$ we add $1 / 2$ times

$\left(\sum_{f \in P_{1}(\rho, k)} a_{1}\left(C_{f}\right)^{m_{1}} \cdot a_{2}\left(C_{f}\right)^{m_{2}} / I_{k}^{\prime}\right) \cdot\left(\sum_{f \in P_{1}(\sigma, k)} a_{1}\left(C_{f}\right)^{n_{1}-m_{1}} \cdot a_{2}\left(C_{f}\right)^{n_{2}-m_{2}} / I_{k}^{\prime}\right)$.

Case ii) If $\rho=\sigma$ and $\left(m_{1}, m_{2}\right)=\left(n_{1}-m_{1}, n_{2}-m_{2}\right)$, we have the contribution from pairs of elliptic curves that are defined over $k$,

$$
1 / 2 \cdot\left(\sum_{f \in P_{1}(\rho, k)} a_{1}\left(C_{f}\right)^{m_{1}} \cdot a_{2}\left(C_{f}\right)^{m_{2}} / I_{k}^{\prime}\right)^{2} .
$$

Moreover, if in addition $n_{1}=0$ and $\nu_{i}=0$ for all odd $i$, then the two elliptic curves together with marked ramification and ordinary points may also form a conjugate pair. We construct these by taking an elliptic curve defined over $k_{2}$, and join it at the origin with its Frobenius conjugate. Define the partition $\nu^{1 / 2}:=\left[1^{\nu_{2}} 2^{\nu_{4}} 3^{\nu_{6}}\right]$. We then add

$$
1 / 2 \cdot\left(\sum_{f \in P_{1}\left(\nu^{1 / 2}, k_{2}\right)} a_{1}\left(C_{f}\right)^{n_{2}} / I_{k_{2}}^{\prime}\right) .
$$

In both these formulas, the factor $1 / 2$ is due to the extra automorphism.

5.3. Adding the contributions from the two strata. To the irreducible representation of $\mathbb{S}_{6}$ indexed by the partition $\mu$ of 6 we can associate $s_{\mu}$, the ordinary Schur polynomial, and to an irreducible representation of the symplectic group $\operatorname{Sp}(4, \mathbb{Q})$ indexed by the partition $\lambda=[l, m]$, the Schur polynomial $s_{<\lambda>}$, see [12, Appendix A]. Written in terms of the power sums $p_{i}$ we have $s_{\mu}=\sum_{\nu} \alpha_{\nu}^{\mu} \cdot p_{1}^{\nu_{1}} \cdots p_{6}^{\nu 6}$ and $s_{<\lambda>}=\sum_{n_{1}, n_{2}} \beta_{n_{1}, n_{2}}^{\lambda} \cdot p_{1}^{n_{1}} p_{2}^{n_{2}}$ for some rational numbers $\alpha_{\nu}^{\mu}$ and $\beta_{n_{1}, n_{2}}^{\lambda}$. The trace of Frobenius on $e_{e t, \mu}\left(\mathcal{A}_{2}[2] \otimes\right.$ $\overline{\mathbb{F}}_{q}, \mathbb{V}_{\lambda}$ ) is then equal to (compare [2, Eq. (3.1)] or [4, Sec. 4.2])

$$
\sum_{n_{1}, n_{2}} \sum_{\nu} \alpha_{\nu}^{\mu} \beta_{n_{1}, n_{2}}^{\lambda} \cdot\left(a\left(\mathcal{M}_{2}, \nu, n_{1}, n_{2}\right)+a\left(\mathcal{A}_{1,1}, \nu, n_{1}, n_{2}\right)\right) \cdot q^{\frac{|\lambda|-n_{1}-n_{2}}{2}} .
$$

Using these results we have been able to (conjecturally) identify the nonEisenstein pieces of $e_{c}\left(\mathcal{A}_{2}[2], \mathbb{V}_{l, m}\right)$. In this process we have greatly benefited from William Stein's tables of modular forms [21].

\section{The Strict Endoscopic Part}

The Hecke algebra of $\operatorname{GSp}(4, \mathbb{Q})$ acts on the inner cohomology of the local system $\mathbb{V}_{l, m}$, cf. e.g. [17, [10], p. 249 and [22]. This inner cohomology on $\mathcal{A}_{2}\left(w^{n}\right)$ also has a Hodge filtration $(0) \subseteq F^{l+m+3} \subseteq F^{l+2} \subseteq F^{m+1} \subseteq F^{0}$. The action of the Hecke algebra respects this Hodge filtration. We now consider the irreducible representations $H$ of the Hecke algebra occurring in the inner cohomology which have the property that $F^{l+m+3} \cap H=(0)$. We define the strict endoscopic part of the inner cohomology of our local system $\mathbb{V}_{l, m}$ on $\mathcal{A}_{2}\left(w^{n}\right)$ to be the direct sum of the representations with that property, i.e., the part that contains no contribution from holomorphic (vector-valued) Siegel modular cusp forms of weight $(j, k)=(l-m, m+3)$. 
Here we assume that $h^{l+m+3,0}=h^{0, l+m+3}$, which follows from the fact that eigenforms have totally real eigenvalues and all representations of $\operatorname{GSp}\left(4, \mathbb{F}_{2}\right)$ are defined over a totally real field, or even $\mathbb{Q}$. This endoscopic part should come from the group $\mathrm{GL}(2, \mathbb{Q}) \times \mathrm{GL}(2, \mathbb{Q}) / \mathbb{G}_{m}$.

Assuming that one has a motive $S\left[\Gamma_{2}[2],(j, k)\right]$ associated to the space $S_{j, k}\left(\Gamma_{2}[2]\right)$ of Siegel modular forms, or more mildly, that one can identify the corresponding part (of rank $4 \operatorname{dim} S_{l-m, m+3}\left(\Gamma_{2}[2]\right)$ ) in the inner cohomology of $\mathbb{V}_{l, m}$ on $\mathcal{A}_{2}[2]$, one might define the strict endoscopic part of the inner cohomology by the equation

$$
e_{\mathrm{End}^{\mathrm{s}}}\left(\mathcal{A}, \mathbb{V}_{l, m}\right):=e_{c}\left(\mathcal{A}, \mathbb{V}_{l, m}\right)-e_{\mathrm{Eis}}\left(\mathcal{A}, \mathbb{V}_{l, m}\right)+S\left[\Gamma_{2}[2],(l-m, m+3)\right] .
$$

Remark 6.1. In the case of a Saito-Kurokawa lift of weight $(0, k)$ the associated rank 4 motive is $S[S L(2, \mathbb{Z}), 2 k-2]+L^{k-1}+L^{k-2}$.

Using our counts of curves over finite fields with marked Weierstrass points and our formulas for the Eisenstein cohomology, we are able to make precise conjectures on the strict endoscopic part. This part of the cohomology is describable in terms of elliptic modular forms (but note that there are also other parts that are describable in terms of such forms).

Conjecture 6.2. Let $k:=l+m+4, k^{\prime}:=l-m+2$, then the strict endoscopic part of the inner cohomology of $\mathbb{V}_{l, m}$ on $\mathcal{A}_{2}[2]$ is given by

$$
-5 L^{m+1} \cdot \operatorname{dim} S_{k}\left(\Gamma_{0}(4)\right) \cdot S\left[\Gamma_{0}(4), k^{\prime}\right],
$$

where we interpret $S\left[\Gamma_{0}(4), 2\right]$ as $-L-1$.

\section{A lifting to Vector-Valued Modular Forms}

The Saito-Kurokawa lifting (see e.g. [18, 24]) gives a way to transform a cusp form $f$ that is a normalized eigenform of weight $2 k$ ( $k$ odd) on $\operatorname{SL}(2, \mathbb{Z})$ into a scalar-valued cusp (eigen) form of weight $k+1$ on $\operatorname{Sp}(4, \mathbb{Z})$. In terms of Galois representations or $L$-factors, the reciprocal of the characteristic polynomial of Frobenius at a prime $p$ is in the Saito-Kurokawa case equal to

$$
\left(1-p^{k-1} X\right)\left(1-a(p) X+p^{2 k-1} X^{2}\right)\left(1-p^{k} X\right),
$$

with $a(p)$ the Hecke eigenvalue of $f$ at $p$.

Based on our calculations of the cohomology of local systems $\mathbb{V}_{l, m}$ on $\mathcal{A}_{2}[2]$, we conjecture the following (Yoshida type) lifting from pairs of elliptic modular forms to vector-valued Siegel modular forms. Recall the notion of spinor $L$-function (see [1]) and that the Atkin-Lehner involution $w_{2}$ acts on $S_{k}\left(\Gamma_{0}(2)\right)$ with eigenspaces $S_{k}^{+}\left(\Gamma_{0}(2)\right)$ and $S_{k}^{-}\left(\Gamma_{0}(2)\right)$ for the eigenvalues +1 and -1 .

Conjecture 7.1. For an eigenform $f \in S_{l+m+4}\left(\Gamma_{0}(2)\right)^{\text {new }}$ and an eigenform $g \in S_{l-m+2}\left(\Gamma_{0}(2)\right)^{\text {new }}$ there is a Siegel modular form $F \in S_{l-m, m+3}\left(\Gamma_{2}[2]\right)$, an eigenform for the Hecke algebra, with spinor L-function

$$
L(F, s)=L(f, s) L(g, s-m-1) .
$$


The form $F$ will appear with multiplicity 5 in $S_{l-m, m+3}\left(\Gamma_{2}[2]\right)$ if $f$ and $g$ have the same eigenvalue \pm under $w_{2}$ and with multiplicity 1 if they have opposite eigenvalues under $w_{2}$.

Similarly, for an eigenform $f \in S_{l+m+4}\left(\Gamma_{0}(4)\right)^{\text {new }}$ and an eigenform $g \in$ $S_{l-m+2}\left(\Gamma_{0}(4)\right)^{\text {new }}$ there is a Siegel modular form $F \in S_{l-m, m+3}\left(\Gamma_{2}[2]\right)$ with spinor L-function

$$
L(F, s)=L(f, s) L(g, s-m-1)
$$

and it will appear with multiplicity 5 in $S_{l-m, m+3}\left(\Gamma_{2}[2]\right)$.

Remark 7.2. The first part of this conjecture is consistent with work of Böcherer and Schulze-Pillot who constructed a Yoshida-type lifting for a pair $(f, g)$ of newforms on $\Gamma_{0}(2)$ with the same sign under $w_{2}$ to Siegel modular forms on the congruence subgroup $\Gamma_{0}^{(2)}(2) \subset \mathrm{Sp}(4, \mathbb{Z})$; see [6], Thm. 5.1 and the ensuing remark on p. 99.

Note that by Tsushima (see [23]) we know the dimensions of the spaces $S_{j, k}\left(\Gamma_{2}[2]\right)$ of Siegel modular forms of weight $(j, k)$ on the group $\Gamma_{2}[2]$. In the cases $(j, k)=(4,4),(6,3)$ and $(8,3)$ it seems that the conjectured lifts generate all of $S_{j, k}\left(\Gamma_{2}[2]\right)$.

Let us define $S_{j, k}^{\text {lift }}\left(\Gamma_{2}[2]\right)$ to be the subspace in $S_{j, k}\left(\Gamma_{2}[2]\right)$ consisting of the cusp forms obtained by the lifting described above. The following conjecture tells us the action of $\mathbb{S}_{6}$ on the space of lifted cusp forms.

Notation 7.3. Put $\tau_{k}^{+}:=\operatorname{dim} S_{k}^{+}\left(\Gamma_{0}(2)\right)^{\text {new }}$ and $\tau_{k}^{-}:=\operatorname{dim} S_{k}^{-}\left(\Gamma_{0}(2)\right)^{\text {new }}$.

Conjecture 7.4. If we assume that $l \neq m$ and let $k:=l+m+4, k^{\prime}:=$ $l-m+2$, then $S_{l-m, m+3}^{\text {lift }}\left(\Gamma_{2}[2]\right)$ decomposes as a representation of $\mathbb{S}_{6}$ as

$$
\begin{aligned}
& s\left[2,1^{4}\right]^{\oplus \tau_{4, k^{\prime}}} \otimes S_{k}\left(\Gamma_{0}(4)\right)^{\text {new }} \oplus\left(s\left[2^{3}\right]^{\oplus \tau_{k^{\prime}}^{+}} \oplus s\left[1^{6}\right]^{\oplus \tau_{k^{\prime}}^{-}}\right) \otimes S_{k}^{+}\left(\Gamma_{0}(2)\right)^{\text {new }} \oplus \\
& \left(s\left[2^{3}\right]^{\oplus \tau_{k^{\prime}}^{-}} \oplus s\left[1^{6}\right]^{\oplus \tau_{k^{\prime}}^{+}}\right) \otimes S_{k}^{-}\left(\Gamma_{0}(2)\right)^{\text {new }} .
\end{aligned}
$$

Remark 7.5. Note that there are no vector-valued lifts of level 1.

We also give a corresponding conjecture for the Saito-Kurokawa lifts.

Conjecture 7.6. If we assume that $l=m$ and let $k:=l+m+4$, then $S_{l-m, m+3}^{\mathrm{lift}}\left(\Gamma_{2}[2]\right)$ decomposes as a representation of $\mathbb{S}_{6}$ for $m$ odd as $s[4,2] \otimes S_{k}^{+}\left(\Gamma_{0}(2)\right)^{\text {new }} \oplus s\left[2^{3}\right] \otimes S_{k}^{-}\left(\Gamma_{0}(2)\right)^{\text {new }} \oplus\left(s[6] \oplus s[4,2] \oplus s\left[2^{3}\right]\right) \otimes S_{k}\left(\Gamma_{0}(1)\right)$, and for $m$ even as

$$
s\left[3^{2}\right] \otimes S_{k}\left(\Gamma_{0}(4)\right)^{\text {new }} \oplus s[5,1] \otimes S_{k}^{-}\left(\Gamma_{0}(2)\right)^{\text {new }} \oplus s\left[1^{6}\right] \otimes S_{k}^{+}\left(\Gamma_{0}(2)\right)^{\text {new }} .
$$

\section{DeComposing the ENDOSCOPIC CONTRIBUtion}

The Saito-Kurokawa lift for level 1 associates, for odd $l=m$, to the space $S_{l+m+4}(\mathrm{SL}(2, \mathbb{Z}))$ of cusp forms on $\mathrm{SL}(2, \mathbb{Z})$ the motive

$$
-S[\mathrm{SL}(2, \mathbb{Z}), l+m+4]-s_{l+m+4}\left(L^{l+2}+L^{m+1}\right)
$$


in the cohomology of the local system $\mathbb{V}_{l, m}$ on $\mathcal{A}_{2}$, the minus sign indicating that it lands in odd degree cohomology. In Conjecture 4.1 of [11] we conjecture the existence of a (strict) endoscopic part

$$
-s_{l+m+4} S[\mathrm{SL}(2, \mathbb{Z}), l-m+2] L^{m+1}=s_{l+m+4}\left(L^{l+2}+L^{m+1}\right) .
$$

Assuming this and adding the two contributions the net result would be the existence of $S[\operatorname{SL}(2, \mathbb{Z}), l+m+4]$ in the inner cohomology. In level 2 we see a similar phenomenon that becomes clearer if one takes the action of $\mathbb{S}_{6}$ into account. So it makes sense to add the two contributions under the heading 'expanded endoscopy.'

For $l \neq m$ and $f \in S_{l+m+4}\left(\Gamma_{0}(N)\right)^{\text {new }}$ and $g \in S_{l-m+2}\left(\Gamma_{0}(N)\right)^{\text {new }}$ the motive of the corresponding lifting is of the form $M_{f}+L^{m+1} M_{g}$ where $M_{f}$ and $M_{g}$ denote the motives associated to the cusp forms $f$ and $g$. Let us call $M_{f}$ the 'leading' part of the vector-valued lift and $L^{m+1} M_{g}$ the 'trailing' one. Note that in the cohomology where this lift appears, the trailing part is of precisely the same form as the strict endoscopy. Let us define the expanded endoscopic part of the cohomology to be the strict endoscopy plus the contribution from the trailing terms coming from the lifts described in Conjecture 7.1 .

Conjecture 8.1. Assume that $l \neq m$ and let $k:=l+m+4, k^{\prime}:=l-m+2$. The expanded endoscopic part of the inner cohomology of $\mathbb{V}_{l, m}$ on $\mathcal{A}_{2}[2]$ is given by

$$
\begin{gathered}
-L^{m+1}\left(\left(\tau_{4, k} \cdot s\left[3,1^{3}\right]+\tau_{1, k} \cdot s\left[3^{2}\right]+\left(\tau_{1, k}+\tau_{2, k}\right) \cdot s\left[4,1^{2}\right]\right) S\left[\Gamma_{0}(4), k^{\prime}\right]^{\text {new }}\right. \\
+\left(\left(\tau_{1, k}+\tau_{2, k}\right) \cdot s[3,2,1]+\tau_{4, k} \cdot s\left[4,1^{2}\right]+\tau_{1, k} \cdot s[4,2]+\tau_{1, k} \cdot s[5,1]\right) S\left[\Gamma_{0}(2), k^{\prime}\right]^{\text {new }} \\
+\left(\tau_{k}^{+} \cdot s[4,2]+\tau_{k}^{-} \cdot s[5,1]\right) S^{+}\left[\Gamma_{0}(2), k^{\prime}\right]^{\text {new }} \\
+\left(\tau_{k}^{-} \cdot s[4,2]+\tau_{k}^{+} \cdot s[5,1]\right) S^{-}\left[\Gamma_{0}(2), k^{\prime}\right]^{\text {new }} \\
+\left(\tau_{1, k} \cdot s\left[2^{3}\right]+\left(\tau_{1, k}+\tau_{2, k}\right) \cdot s[3,2,1]+\tau_{4, k} \cdot s\left[3^{2}\right]+\tau_{4, k} \cdot s\left[4,1^{2}\right]+\left(\tau_{2, k}+2 \tau_{1, k}\right) \cdot s[4,2]\right. \\
\left.\left.+\left(\tau_{1, k}+\tau_{2, k}\right) \cdot s[5,1]+\tau_{1, k} \cdot s[6]\right) S\left[\Gamma_{0}(1), k^{\prime}\right]^{\text {new }}\right) .
\end{gathered}
$$

Remark 8.2. In Conjecture 8.1 both the strict endoscopy and the lifts from Conjecture 7.1 contribute to the terms $-L^{m+1} \cdot \tau_{4, k} \cdot s\left[3,1^{3}\right] S\left[\Gamma_{0}(4), k^{\prime}\right]^{\text {new }}$, $-L^{m+1}\left(\tau_{k}^{+} \cdot s[4,2]+\tau_{k}^{-} \cdot s[5,1]\right) S^{+}\left[\Gamma_{0}(2), k^{\prime}\right]^{\text {new }}$ and $-L^{m+1}\left(\tau_{k}^{-} \cdot s[4,2]+\right.$ $\left.\tau_{k}^{+} \cdot s[5,1]\right) S^{-}\left[\Gamma_{0}(2), k^{\prime}\right]^{\text {new }}$.

Conjecture 8.3. Assume that $l=m$ and let $k:=2 m+4$. The expanded endoscopic part of the inner cohomology of $\mathbb{V}_{m, m}$ on $\mathcal{A}_{2}[2]$ is given by

$$
L^{m+1} \cdot(L+1) \cdot \begin{cases}\tau_{k}^{+} \cdot s\left[1^{6}\right]+\tau_{4, k} \cdot s\left[3^{2}\right]+\tau_{k}^{-} \cdot s[5,1] & \text { if } m \text { odd } \\ \left(\tau_{k}^{-}+\tau_{1, k}\right) \cdot s\left[2^{3}\right]+\left(\tau_{k}^{+}+\tau_{1, k}\right) \cdot s[4,2]+\tau_{1, k} \cdot s[6] & \text { if } m \text { even }\end{cases}
$$




\section{Dimension Checks}

In the case of one Weierstrass point we have computed the numerical Euler characteristic $\sum(-1)^{i} \operatorname{dim} H_{c}^{i}\left(\mathcal{A}_{2}\left(w^{1}\right), \mathbb{V}_{l, m}\right) \in \mathbb{Z}$ for any $(l, m)$ using methods as in [3] and [5]. The conjectural results agree for $(l, m)$ with $l+m \leq 10$ with these numerical Euler characteristics of the local systems and moreover for larger values of $(l, m)$, e.g. for $l+m \leq 20$, we find that the numerical Euler characteristic minus the conjectured Eisenstein and endoscopic part, is always a non-positive multiple of 4 . For all $l+m \leq 20$, this number equals -4 times the dimension of the space of Siegel modular cusp forms $S_{l-m, m+3}\left(\Gamma_{2}\left(w^{1}\right)\right)$ as calculated by a program provided to us by R. Tsushima.

\section{Examples of Eigenvalues of Hecke Eigenforms}

We will now give a number of examples. We first write out some (conjectural) results for the first few local systems. Needless to say they are based on ample numerical evidence. Recall that the cohomology has the following parts,

$$
e_{c}\left(\mathcal{A}, \mathbb{V}_{l, m}\right)=e_{\text {Eis }}\left(\mathcal{A}, \mathbb{V}_{l, m}\right)+e_{\operatorname{End}^{\mathrm{s}}}\left(\mathcal{A}, \mathbb{V}_{l, m}\right)-S\left[\Gamma_{2}[2],(l-m, m+3)\right] .
$$

Here we will write $\Phi_{N, k}:=S\left[\Gamma_{0}(N), k\right]^{\text {new }}$ and in all of the following cases this will be a motive associated to a single newform.

\begin{tabular}{|r|r|}
\hline$(l, m)$ & $e_{c}\left(\mathcal{A}_{2}[2], \mathbb{V}_{l, m}\right)$ \\
\hline$(0,0)$ & $L^{3}+L^{2}-14 L+16$ \\
$(2,0)$ & $-30 L+30$ \\
$(1,1)$ & $5 L^{3}-10 L^{2}$ \\
$(4,0)$ & $-45 L+45-10 L \Phi_{4,6}$ \\
$(3,1)$ & $-30 L^{2}-15 \Phi_{4,6}$ \\
$(2,2)$ & $9 L^{4}-21 L^{3}-\Phi_{2,8}$ \\
$(6,0)$ & $-60 L+60-31 L \Phi_{2,8}-\Phi_{2,10}$ \\
$(5,1)$ & $-45 L^{2}+15-30 \Phi_{2,8}-20 L \Phi_{4,6}-5 \Phi_{4,10}$ \\
$(4,2)$ & $-45 L^{3}+45-S\left[\Gamma_{2}[2],(2,5)\right]$ \\
$(3,3)$ & $10 L^{5}-35 L^{4}-15 \Phi_{4,6}-5 \Phi_{2,10}$ \\
$(8,0)$ & $-75 L+75-25 L \Phi_{4,10}-40 L \Phi_{2,10}-5 \Phi_{4,12}$ \\
$(7,1)$ & $-60 L^{2}+30-15 \Phi_{4,10}-30 \Phi_{2,10}-40 L^{2} \Phi_{2,8}-S\left[\Gamma_{2}[2],(6,4)\right]$ \\
$(6,2)$ & $-60 L^{3}+60-20 L^{3} \Phi_{4,6}-S\left[\Gamma_{2}[2],(4,5)\right]$ \\
$(5,3)$ & $-60 L^{4}-30 \Phi_{2,8}-S\left[\Gamma_{2}[2],(2,6)\right]$ \\
$(4,4)$ & $15 L^{6}-45 L^{5}+30-15 \Phi_{4,6}-5 \Phi_{4,12}$ \\
\hline
\end{tabular}

In a number of cases, we can identify "genuine" Siegel modular forms, i.e. not lifts of the type described in Conjecture 7.1. The space $S_{j, k}\left(\Gamma_{2}[2]\right)$, and therefore also the motive (or corresponding part of the cohomology) $S\left[\Gamma_{2}[2],(j, k)\right]$ can be decomposed under the action of $\mathbb{S}_{6}$ into a sum of spaces 
$S_{j, k}\left(\Gamma_{2}[2]\right)^{\mu}$ corresponding to the $\mathbb{S}_{6}$-representation given by the partition $\mu$. For the cases appearing in the table above we have

$$
\begin{gathered}
S_{2,5}\left(\Gamma_{2}[2]\right)=S_{2,5}\left(\Gamma_{2}[2]\right)^{\left[2^{2}, 1^{2}\right]} \\
S_{6,4}\left(\Gamma_{2}[2]\right)=S_{6,4}\left(\Gamma_{2}[2]\right)^{\left[2^{2}, 1^{2}\right]} \oplus S_{6,4}\left(\Gamma_{2}[2]\right)^{\left[3,1^{3}\right]} \\
S_{4,5}\left(\Gamma_{2}[2]\right)=S_{4,5}\left(\Gamma_{2}[2]\right)^{\left[2,1^{4}\right]} \oplus S_{4,5}\left(\Gamma_{2}[2]\right)^{\left[2^{2}, 1^{2}\right]} \oplus S_{4,5}\left(\Gamma_{2}[2]\right)^{[3,2,1]} \\
S_{2,6}\left(\Gamma_{2}[2]\right)=S_{2,6}\left(\Gamma_{2}[2]\right)^{\left[3,1^{3}\right]} \oplus S_{2,6}\left(\Gamma_{2}[2]\right)^{[3,2,1]},
\end{gathered}
$$

and each of these subspaces is generated by one vector-valued Siegel modular form. For instance for $(l, m)=(4,2)$ we have one vector-valued Siegel modular form appearing with the representation $s\left[2^{2}, 1^{2}\right]$, i.e. with multiplicity 9 , which agrees with the result of Tsushima that $S_{2,5}\left(\Gamma_{2}[2]\right)$ is 9-dimensional, see [23]. Moreover, according to our data $S_{4,5}\left(\Gamma_{2}[2]\right)^{\left[2,1^{4}\right]}$ is generated by a lift with corresponding motive $\Phi_{4,12}+L^{3} \Phi_{4,6}$.

The trace of Frobenius, for a prime $p>2$, on the space $S\left[\Gamma_{2}[2],(j, k)\right]$ is equal to the trace of the Hecke operator $T(p)$ on $S_{j, k}\left(\Gamma_{2}[2]\right)$. In the following two tables we write the (conjectural) Hecke eigenvalues for the generating Siegel modular form for $3 \leq p \leq 19$ in four cases when $S_{j, k}\left(\Gamma_{2}[2]\right)^{\mu}$ is generated by a single vector-valued Siegel modular form. We are assuming here the conjectures on the endoscopy given above. Note that all these eigenvalues have many small prime factors.

\begin{tabular}{|c|c|c|}
\hline$p$ & $S_{2,5}\left(\Gamma_{2}[2]\right)^{\left[2^{2}, 1^{2}\right]}$ & $S_{6,4}\left(\Gamma_{2}[2]\right)^{\left[2^{2}, 1^{2}\right]}$ \\
\hline 3 & $-2^{3} \cdot 5$ & $-2^{3} \cdot 5 \cdot 7$ \\
5 & $-2^{2} \cdot 5^{2} \cdot 13$ & $-2^{2} \cdot 5 \cdot 149$ \\
7 & $2^{4} \cdot 3 \cdot 5 \cdot 13$ & $-2^{4} \cdot 3 \cdot 5 \cdot 401$ \\
11 & $2^{3} \cdot 11 \cdot 13 \cdot 31$ & $2^{3} \cdot 36383$ \\
13 & $-2^{2} \cdot 5 \cdot 3469$ & $2^{2} \cdot 5 \cdot 37 \cdot 251$ \\
15 & $-2^{2} \cdot 5 \cdot 11 \cdot 13 \cdot 197$ & $2^{2} \cdot 5 \cdot 19 \cdot 6983$ \\
17 & $2^{3} \cdot 5^{2} \cdot 11 \cdot 13 \cdot 17$ & $-2^{3} \cdot 5 \cdot 29 \cdot 6287$ \\
19 & $-2^{4} \cdot 5 \cdot 13 \cdot 311$ & $-2^{4} \cdot 5 \cdot 43 \cdot 2267$ \\
\hline
\end{tabular}

\begin{tabular}{|c|c|c|}
\hline$p$ & $S_{6,4}\left(\Gamma_{2}[2]\right)^{\left[3,1^{3}\right]}$ & $S_{10,3}\left(\Gamma_{2}[2]\right)^{\left[2^{2}, 1^{2}\right]}$ \\
\hline 3 & $-2^{3} \cdot 3$ & $2^{3} \cdot 5^{2}$ \\
5 & $2^{2} \cdot 3^{2} \cdot 7 \cdot 41$ & $2^{2} \cdot 5 \cdot 127$ \\
7 & $2^{4} \cdot 5^{2} \cdot 73$ & $-2^{4} \cdot 3 \cdot 5^{2} \cdot 13$ \\
11 & $-2^{3} \cdot 3^{2} \cdot 4793$ & $-2^{3} \cdot 439 \cdot 1123$ \\
13 & $-2^{2} \cdot 7 \cdot 21563$ & $2^{2} \cdot 5^{2} \cdot 47 \cdot 4457$ \\
15 & $-2^{2} \cdot 3^{2} \cdot 2351$ & $2^{2} \cdot 5^{2} \cdot 799441$ \\
17 & $-2^{3} \cdot 7 \cdot 11 \cdot 37 \cdot 383$ & $2^{3} \cdot 5 \cdot 7 \cdot 461 \cdot 1723$ \\
19 & $-2^{4} \cdot 3^{2} \cdot 11 \cdot 17 \cdot 29 \cdot 43$ & $2^{4} \cdot 5^{2} \cdot 3653483$ \\
\hline
\end{tabular}


We compute the slopes for the single Siegel modular cusp form generating the space $S_{2,6}\left(\Gamma_{2}[2]\right)^{\left[3,1^{3}\right]}$ and for the one generating $S_{2,6}\left(\Gamma_{2}[2]\right)^{[3,2,1]}$. Recall that the reciprocal of the characteristic polynomial of Frobenius is

$$
1-\lambda(p) X+\left(\lambda(p)^{2}-\lambda\left(p^{2}\right)-p^{l+m+2}\right) X^{2}-\lambda(p) p^{l+m+3} X^{3}+p^{2 l+2 m+6} X^{4}
$$

and the 'slope' refers to the slopes of the Newton polygon.

\begin{tabular}{|c|c|c|c|}
\hline$p$ & $\lambda(p)$ & $\lambda\left(p^{2}\right)$ & slopes \\
\hline 3 & $2^{3} \cdot 3^{3}$ & $-2^{2} \cdot 3^{6} \cdot 107$ & $3,3,8,8$ \\
5 & $-2^{2} \cdot 3^{4} \cdot 17$ & $2^{2} \cdot 181 \cdot 26161$ & $11 / 2$ \\
\hline \hline 3 & $-2^{3} \cdot 3^{2} \cdot 5$ & $3^{4} \cdot 1753$ & $2,2,9,9$ \\
5 & $2^{2} \cdot 3 \cdot 5 \cdot 7^{2}$ & $5^{2} \cdot 117119$ & $1,1,10,10$ \\
\hline
\end{tabular}

\section{HARDER'S CONGRUENCES}

Harder predicts a congruence between an elliptic modular form $f$ and a Siegel modular form whenever a 'large' prime $\ell$ divides a critical value $L(f, s)$ of the $L$-series of the elliptic modular form, see [17, 13]. In cooperation with Harder we checked a few cases. This lends at the same time credibility to our computations and conjectures and to Harder's conjecture.

For example, if $f$ is a newform in the 1-dimensional space $S_{20}^{+}\left(\Gamma_{0}(2)\right)$ then 61 divides $L(f, 12)$ and one expects the congruence

$$
p^{8}+a(p)+p^{11} \equiv \lambda(p)(\bmod 61)
$$

for the Hecke eigenvalues $\lambda(p)$ of an eigenform $F \in S_{2,10}\left(\Gamma_{2}[2]\right)$ for every prime $p \neq 2$. By using dimension formulas of R. Tsushima for $S_{j, k}\left(\Gamma_{2}\left(w^{0}\right)\right)$ and $S_{j, k}\left(\Gamma_{2}\left(w^{1}\right)\right)$ (see also Section 9) we find that $\operatorname{dim} S_{2,10}\left(\Gamma_{2}\left(w^{0}\right)\right)=0$ and $\operatorname{dim} S_{2,10}\left(\Gamma_{2}\left(w^{1}\right)\right)=1$. For $p \leq 37$ the eigenvalues $\lambda(p)$ we have calculated for a non-zero $F \in S_{2,10}\left(\Gamma_{2}\left(w^{1}\right)\right)$ satisfy the required congruence, e.g. $\lambda(3)=$ 18360 and $3^{8}-13092+3^{11} \equiv 18360(\bmod 61)$.

In the following table we list a few congruences that are valid for the eigenvalues that we find. Also in these cases the corresponding spaces of modular forms are 1-dimensional and the Siegel modular forms do not come from level 1.

\begin{tabular}{|c|c|c|c|}
\hline$\langle f\rangle$ & $\langle F\rangle$ & $s$ & $\ell$ \\
\hline$S_{20}^{+}\left(\Gamma_{0}(2)\right)$ & $S_{2,10}\left(\Gamma_{2}\left(w^{1}\right)\right)$ & 12 & 61 \\
$S_{20}^{+}\left(\Gamma_{0}(2)\right)$ & $S_{10,6}\left(\Gamma_{2}\left(w^{1}\right)\right)$ & 16 & 109 \\
$S_{18}^{-}\left(\Gamma_{0}(2)\right)$ & $S_{6,7}\left(\Gamma_{2}\left(w^{1}\right)\right)$ & 13 & 29 \\
$S_{20}^{-}\left(\Gamma_{0}(2)\right)$ & $S_{12,5}\left(\Gamma_{2}\left(w^{1}\right)\right)$ & 17 & 79 \\
$S_{22}^{+}\left(\Gamma_{0}(2)\right)$ & $S_{16,4}\left(\Gamma_{2}\left(w^{1}\right)\right)$ & 20 & 37 \\
\hline
\end{tabular}


Moreover, for a newform $f \in S_{16}\left(\Gamma_{0}(4)\right)$ we find that our traces of Frobenius on both $S_{8,5}\left(\Gamma_{2}[2]\right)^{\left[4,1^{2}\right]}$ and $S_{8,5}\left(\Gamma_{2}[2]\right)^{\left[3^{2}\right]}$, satisfy the expected congruence modulo $\ell=37$, which divides $L(f, 13)$.

\section{REFERENCES}

[1] A.N. Andrianov: Euler products that correspond to Siegel modular forms of genus 2. Uspehi Mat. Nauk 29 (3(177)) (1974), 43-110. English transl.: Russ. Math. Surv. 29 (1974).

[2] J. Bergström: Equivariant counts of points of the moduli spaces of pointed hyperelliptic curves, available at arxiv:math. AG/0611813.

[3] J. Bergström, G. van der Geer: The Euler characteristic of local systems on the moduli of curves and abelian varieties of genus three. arXiv:0705.0293

[4] J. Bergström, O. Tommasi: The rational cohomology of $\overline{\mathcal{M}}_{4}$. Math. Ann. 338 (2007), no. 1, 207-239.

[5] G. Bini, G. van der Geer: The Euler characteristic of local systems on the moduli of genus 3 hyperelliptic curves, Math. Ann. 332 (2005), 367-379.

[6] S. Böcherer, R. Schulze-Pillot: Siegel modular forms and theta series attached to quaternion algebras. Nagoya Math. J. 147 (1997), 71-106.

[7] C. Consani, C. Faber: On the cusp form motives in genus 1 and level 1. Moduli spaces and arithmetic geometry, 297-314, Adv. Stud. Pure Math., 45, Math. Soc. Japan, Tokyo, 2006.

[8] P. Deligne: Formes modulaires et représentations $\ell$-adiques, Séminaire Bourbaki 19681969, exp. 355.

[9] G. Faltings: On the cohomology of locally symmetric Hermitian spaces. Paul Dubreil and Marie-Paule Malliavin algebra seminar, 35th year (Paris, 1982), p. 55-98, Lecture Notes in Math., 1029, Springer, Berlin, 1983.

[10] G. Faltings, C-L. Chai: Degeneration of abelian varieties. Ergebnisse der Mathematik 22, Springer Verlag.

[11] C. Faber, G. van der Geer: Sur la cohomologie des systèmes locaux sur les espaces des modules des courbes de genre 2 et des surfaces abéliennes, I, II. C.R. Acad. Sci. Paris, Sér. I, 338 (2004), 381-384, 467-470.

[12] W. Fulton, J. Harris: Representation Theory. A First Course, Springer-Verlag, New York, 1991.

[13] G. van der Geer: Siegel modular forms and their applications. In: J. Bruinier, G. van der Geer, G. Harder, D. Zagier: The 1-2-3 of modular forms. Springer Verlag.

[14] G. van der Geer: Rank one Eisenstein cohomology of local systems on the moduli space of abelian varieties. arXiv:0802.2921

[15] E. Getzler: Topological recursion relations in genus 2. In: Integrable systems and algebraic geometry (Kobe/Kyoto, 1997), 73-106. World Sci. Publishing, River Edge.

[16] G. Harder: A congruence between a Siegel and an elliptic modular form. In: J. Bruinier, G. van der Geer, G. Harder, D. Zagier: The 1-2-3 of modular forms. Springer Verlag.

[17] G. Harder: Eisensteinkohomologie und die Konstruktion gemischter Motive. Lecture Notes in Mathematics, 1562. Springer-Verlag, Berlin, 1993.

[18] N. Kurokawa: Examples of eigenvalues of Hecke operators on Siegel cusp forms of degree two. Invent. Math. 49 (1978), no. 2, 149-165.

[19] C. Peters: Lowest Weights in Cohomology of Variations of Hodge Structure. arXiv:0708.0130

[20] A.J. Scholl: Motives for modular forms. Invent. Math. 100 (1990), no. 2, 419-430.

[21] W. Stein: http://modular.fas.harvard.edu/tables/

[22] R. Taylor: On the $\ell$-adic cohomology of Siegel threefolds. Invent. Math. 114 (1993), p. 289-310. 
[23] R. Tsushima: An explicit dimension formula for the spaces of generalized automorphic forms with respect to $\operatorname{Sp}(2, \mathbb{Z})$. Proc. Japan Acad. Ser. A Math. Sci. 59 (1983), no. 4, 139-142.

[24] D. Zagier: Sur la conjecture de Saito-Kurokawa (d'après H. Maass). Seminar on Number Theory, Paris 1979-80, pp. 371-394, Progr. Math., 12, Birkhäuser, Boston, Mass., 1981.

Korteweg-de Vries Institudt, Universiteit van Amsterdam, Plantage Muidergracht 24, 1018 TV Amsterdam, The Netherlands.

E-mail address: bergstro@science.uva.nl

Institutionen för Matematik, Kungliga Tekniska Högskolan, 10044 StockHOLM, SwEDEN.

E-mail address: faber@math.kth.se

Korteweg-de Vries Institudt, Universiteit van Amsterdam, Plantage Muidergracht 24, 1018 TV Amsterdam, The Netherlands.

E-mail address: geer@science.uva.nl 